The Astrophysical Journal, $411:$ L59-L62, 1993 July 10

(C) 1993. The American Astronomical Society. All rights reserved. Printed in U.S.A.

\title{
THE INFLUENCE OF QSO EMISSION-LINE VELOCITY SHIFTS ON ESTIMATES OF THE INTERGALACTIC BACKGROUND INTENSITY
}

\author{
BRIAN R. ESPEY \\ Department of Physics and Astronomy, University of Pittsburgh, Pittsburgh, PA 15260; e-mail: Internet: espey@phyast.pitt.edu \\ Received 1993 February 8; accepted 1993 April 27
}

\begin{abstract}
Observations of the Inverse Effect indicate that the observed number of QSOs cannot provide sufficient radiation to reionize the intergalactic medium. We reanalyze the data set of one such study and show that bias in the measurement of QSO redshifts can account for at least a proportion of the discrepancy between the observed and predicted ionizing background when current QSO luminosity evolution models are used. The exact value of the shortfall depends on the assumed opacity due to intervening absorption systems, but for one optically thick model our reassessment indicates that the apparent discrepancy between the observed ionizing background strength and that predicted from "QSO-only" models is halved. This result places ionization models which solely involve QSO ionizing radiation within a factor of $\approx 4$ of supplying all the photons required by the observations and may be within a factor of 2 if the opacity of the universe has been overestimated in current models. When account is taken of the incompleteness of the QSO luminosity function at high redshift, it is quite possible that the bulk of the intergalactic ionizing radiation is provided by active galactic nuclei.
\end{abstract}

Subject headings: cosmology: observations - quasars: absorption lines - quasars: emission lines ultraviolet: galaxies

\section{INTRODUCTION}

The number of Lya absorbing clouds per unit redshift above a certain minimum cutoff (most commonly an intrinsic equivalent width limit) is observed to increase with increasing redshift when the data from a number of QSOs are considered. In any one object, however, there is a decrease in the number of absorbing systems close to the emission-line redshift of the QSO superposed on this general trend. The decrease in absorbing systems was dubbed the "Inverse Effect" by Murdoch et al. (1986) but has also been called the "Proximity Effect " by other authors. It is generally ascribed to the decreasing fraction of $\mathbb{H}$ I present in otherwise similar intergalactic gas clouds due to photoionization by the QSO's ionizing radiation field. The existence of a photoionization-dependent Inverse Effect provides a method by which the intensity of the intergalactic ionizing background may be measured, as the increased ionization of the gas clouds is due to the extra flux of ionizing photons from the QSO. By determining the radiation intensity from measurements of the QSO's luminosity and the distance between it and each cloud, it is possible to calculate the absolute strength of the UV background (see Lu, Wolfe, \& Turnshek 1991 for a discussion).

Recent measurements made by utilizing the Inverse Effect indicate that the ionizing background is much stronger than the estimated total contribution from QSOs and hence imply that the background must be produced in large part by non-QSO sources (e.g., starburst galaxies) or that the observed number of QSOs is biased to low values through cumulative extinction along our line of sight (Bechtold et al. 1987; Bajtlik, Duncan, \& Ostriker 1988, hereafter BDO; Lu et al. 1991; Fall \& Pei 1993).

We will be concerned in this Letter with the part of the measurement process which concerns the estimation of the intensity of the QSO ionizing flux at the distance of the Ly $\alpha$ cloud. It is important to note two important facts: (1) that it is implicit in the determination of cloud distances that the observed redshift difference between an absorbing cloud and a QSO is solely due to cosmological expansion and (2) that the emission-line redshifts of QSOs used in Inverse Effect studies are generally determined from lines which may be blueshifted relative to the QSO rest frame. Velocity shifts of the order of several thousand kilometers per second occur between emission lines in the broad-line region of active galactic nuclei (AGNs). Because of the small region near a QSO which is influenced by its radiation (less than $8 \mathrm{Mpc}$ or $\approx 52.5 \mathrm{H}_{0} \mathrm{~km}$ $\mathrm{s}^{-1}$ for $\left.q_{0}=0.5 ; \mathrm{BDO}\right)$, the neglect of these velocity differences can cause a serious underestimation of the QSO's sphere of influence and an attendant overestimation of the strength of the ionizing background intensity. We shall estimate the size of this effect by reexamining the absorption-line data of $\mathrm{Lu}$ et al. using a photoionization model consistent with the ionizing background expected for a universe optically thick to ionizing radiation (Madau 1992, hereafter M92).

We shall adopt a cosmology with $H_{0}=50 \mathrm{~km} \mathrm{~s}^{-1} \mathrm{Mpc}^{-1}$ and $q_{0}=0.5$ in what follows.

\section{THE PHOTOIONIZATION MODEL}

In order to estimate the intensity of the UV ionizing background, the observed decrease in the number of Ly $\alpha$ absorption clouds close to a QSO is used to estimate the point at which the QSO radiation ionizes the cloud below the observational detection limit. The intensity of radiation striking an arbitrary Ly $\alpha$ cloud may be represented as

$$
f_{\text {tot }}=f_{B}+f_{\text {QSO }}=(1+\omega) f_{B},
$$

where $f_{\text {tot }}, f_{B}$, and $f_{\text {oso }}$ represent the total, background, and QSO ionizing fluxes, and $\omega$ is a factor to express the QSO's ionizing radiation in terms of the background at that redshift.

To determine how the observed number of Ly $\alpha$ clouds above the detection threshold varies in the neighborhood of a QSO, it 
is necessary to combine the effects of the reaction of a typical cloud to the radiation field with the overall column density distribution of Ly $\alpha$ clouds. The increasing ionization level of an individual cloud is provided by a model, and the distribution of Ly $\alpha$ cloud column densities is obtained from observations of clouds far from local sources of ionization. For isolated Lyo clouds, we adopt $\mathbb{L u}$ et al.'s parameterization of the relationship between the number of clouds, $\mathcal{N}$, and their $\mathbb{H} \mathbb{I}$ column density, $N$, far from local ionizing sources:

$$
d \mathscr{N} / d N \propto N^{-1.7} \text {. }
$$

To determine how the hydrogen ionization fraction varies with the intensity of the radiation striking the cloud $\left(f_{\text {tot }}\right)$, we use the photoionization code CLOUDY 80.06 (Ferland 1991). The clouds are modeled as plane-parallel slabs of primordial abundance gas illuminated by varying combinations of the QSO and background spectra published in M92.

We chose to model a cloud at $z=2.5$, which is close to the median redshift of the Ly $\alpha$ absorption systems in the $\mathbb{L u}$ et al. (1991) sample. A constant density of $10^{-3} \mathrm{~cm}^{-3}$ was used, with the total column density fixed to a value which would provide an $\mathrm{H}$ I column of $10^{13.5} \mathrm{~cm}^{-2}$ for a $\mathrm{Ly} \alpha$ cloud far from local ionization sources. We thus followed previous workers in implicitly assuming that neither mass loss nor expansion takes place as the strength of the ionizing radiation field increases.

The ionization fraction in our model clouds for increasing levels of ionizing radiation was determined for both optically thin and thick models. In the optically thick case this is the first occasion we know of in which the reaction of Ly $\alpha$ clouds to a "realistic" combination of an absorbed ionizing background spectrum and an unabsorbed QSO spectrum has been calculated. Fits to the ionization fractions in clouds at varying distances from the model QSO were used to determine the following relationship:

$$
d \mathscr{N} / d N \propto \begin{cases}(1+\omega)^{-0.5} & \text { (optically thin universe) } \\ (1+\omega)^{-0.7} & \text { (optically thick universe) } .\end{cases}
$$

The response of the clouds in the optically thin and thick cases varies because of the different background spectra. The ionization level of the optically thick clouds closely mimics that expected from hydrogen-only clouds in which the neutral hydrogen fraction varies inversely with the strength of the ionizing radiation field.

\section{VELOCITY SHIFTS IN QSO SAMPLES}

Our aim is to study whether radial motion within the broad emission line region of QSOs can account for at least some of the apparent strength of the ionizing background. Before we do this, however, it is worth reviewing the data concerning velocity shifts which have been obtained to date.

In nearby objects the redshift of the narrow forbidden and broad Balmer lines is in good agreement (i.e., within $100 \mathrm{~km}$ $\mathrm{s}^{-1}$ in the mean) with the systemic value determined from observations of molecular and $\mathbb{H}$ I $21 \mathrm{~cm}$ emission or stellar absorption features (Wilson \& Heckman 1985; Hutchings, Gower, \& Price 1987; Alloin et al. 1992). For more distant objects it becomes progressively more difficult to make observations of the neutral gas and stellar population of the host galaxy, and resort must be had to the secondary indicator provided by the forbidden lines. At intermediate redshifts it is found that the high-ionization lines of $\mathrm{Ly} \alpha$ and $\mathrm{C}$ IV are, in the mean, offset to the blue of the narrow forbidden lines, while the low-ionization lines of $\mathrm{Mg}$ II and the Balmer lines remain in close agreement (Gaskell 1982; Wilkes 1984, 1986; Corbin 1990; Tytler \& Fan 1992). At still higher redshifts ( $z \gtrsim 1$ or so) the strongest forbidden lines lie in the infrared and are thus not easilly measured. Spectra obtained by Carswell et al. (1991) of the luminous QSO MC $1331+170$ support the inferences made from analysis of lower redshift objects by unequivocally showing that the Ly $\alpha$ and C IV lines are blueshifted by $\gtrsim 1200$ $\mathrm{km} \mathrm{s}^{-1}$ from the $[\mathrm{O}$ III $] 5007 \AA$ line, while the $\mathrm{Mg}$ II and Balmer lines are nearly at rest with respect to it.

While the exact value for the mean high-ionization line blueshift in high-redshift QSOs has been the subject of some debate, it appears that determinations of typical values are dependent on sample selection, and a consensus is emerging that larger shifts tend to be found in more luminous objects (Espey 1989; Corbin 1990; Tytler \& Fan 1992). It thus is likely that the very objects used for absorption-line studies are also those which are most severely affected by shifts of their emission lines from the systemic velocity. Analysis of $\mathbb{C}$ IV and $\mathrm{Mg}$ II redshift data from a sample of high-luminosity QSOs indicates that the systemic velocity in the $\mathbb{L u}$ et al. (1991) sample may be offset by $\approx 1450 \mathrm{~km} \mathrm{~s}^{-1}$ to the red of the high-ionization lines (Espey \& Junkkarinen 1993).

The presence of large velocity differences implies that estimates of Ly $\alpha$ cloud distances based on the assumption that the redshift of the $\mathbb{L} y \alpha$ or $\mathbb{C}$ IV line is purely cosmological will result in an underestimation of the distance of the absorbing gas from the QSO. The net result will therefore be an underestimation of the influence of the QSO's ionizing field and a resultant overestimation of the strength of the intergalactic background.

\section{ESTIMATES OF THE IONIZING BACKGROUND}

In what follows we shall concentrate on a universe which is optically thick to ionizing photons, since this is the case where the apparent disagreement between observation and the theoretical QSO background is most severe.

\subsection{The Case of No Redshift Bias}

Our analysis of the absorption-line data to determine the strength of the ionizing background followed the procedures used in previous studies (see, e.g., BDO; Lu et al. 1991). Briefly, the absorption-line data are mapped into a space in which the ordinate is related to the relative intensities of the background and QSO ionizing radiation (specifically, versus $1+\omega$; see eq. [1]). The data are then binned and fitted with a curve of the form shown in equation (3) by shifting the theoretical curve along the $x$-axis until the $\chi^{2}$ of the fit is minimized. The offset required to bring the model curve into agreement with the binned data gives the amount by which the background estimate must be increased to fit the observations.

If we assume that the measured redshifts of the highionization lines of $\mathbb{L y} \alpha$ and $\mathbb{C}$ IV accurately represent the systemic redshift, we determine the intergalactic ionizing background to be $\log J_{v}=-20.8 \pm 0.3(90 \%$ confidence limits), where $J_{v}$ is given in units of ergs $\mathrm{cm}^{-2} \mathrm{~s}^{-1} \mathrm{~Hz}^{-1} \mathrm{sr}^{-1}$ and refers to the mean intensity at the Lyman limit. This value is in good agreement with $\mathrm{Lu}$ et al.'s value of $-21.0 \pm 0.5$. A similar result $\left(\log J_{v}=-21.0 \pm 0.3\right)$ is obtained for the optically thin case with the appropriate choice of fitting function from equation (3), together with Madau's optically thin background model. As has been discussed previously, a value this high cannot be produced if QSOs are the sole sources of ion- 
ization. In the case of Madau's model of an optically thick universe, QSOs can provide only $\approx 7 \%$ of the ionizing photons required, even under the assumption that their number density is constant from a turn-on at $z=5$ (M92).

\subsection{Background Estimates in the Presence of Redshift Biases}

To determine the effect of biases in the QSO redshift, we reanalyzed the Lu et al. (1991) data set under differing assumptions for the velocity shift between the emission-line redshift and the systemic velocity. This was done by raising the emission-line redshift of every QSO in the sample by a fixed velocity and then rebinning and refitting the absorption-line data. The absorption-line data of $\mathbb{L} u$ et al. were complete up to the emission-line redshift for each object quoted in their Table 1. We have used these same values as upper limits to the redshift range for each object when estimating the expected number of absorbing systems. This guards against deriving a lower-than-expected number of observed systems close to each QSO and hence against underestimating the background intensity. We repeated the analysis for a number of velocity shifts in the range $0 \mathrm{~km} \mathrm{~s}^{-1} \lesssim \Delta v \lesssim 2400 \mathrm{~km} \mathrm{~s}^{-1}$ to study the sensitivity of the derived background intensity to biases in the emission-line redshift of the QSO sample.

The background estimates derived from this analysis together with formal $90 \%$ confidence limits derived from $\chi^{2}$ fitting are given in Table 1 and plotted in Figure 1. For the mean shift of $1450 \mathrm{~km} \mathrm{~s}^{-1}$ expected from similarly luminous objects the true value of the intergalactic background radiation is $\log J_{v}=-21.3$. This is within a factor of $\approx 2-3$ of that expected for an optically thick universe in which QSOs provide the sole source of ionization. Furthermore, because of the exponential dependence of the derived $J_{v}$ values on velocity shift, the determined background strength is not overly sensitive to the exact value chosen for the mean emission-line shift for values above $\approx 1000 \mathrm{~km} \mathrm{~s}^{-1}$. As an example, for values of $800 \mathrm{~km} \mathrm{~s}^{-1} \lesssim \Delta v \lesssim 2400 \mathrm{~km} \mathrm{~s}^{-1}$, the derived logarithm of the background intensity varies by less than \pm 0.20 relative to the "best-guess" value of $\log J_{v}=-21.3$, and this is within the $90 \%$ confidence limits for that value.

We have tested the sensitivity of this result to the exponent of the fitting function given in equation (3). Such a change may be due, for instance, to an error in either the power-law

TABLE 1

DEPENDENCE OF IONIZING BACKGROUND STRENGTH ON VELOCITY OFFSET

\begin{tabular}{|c|c|c|}
\hline$\Delta v^{\mathrm{a}}$ & $\log J_{v}^{2 \mathrm{~b}}$ & Error $^{\mathrm{c}}$ \\
\hline $0 \ldots$ & -20.76 & $-0.34,+0.31$ \\
\hline $400 \ldots$ & -21.01 & $-0.25,+0.22$ \\
\hline .......... & -21.11 & $-0.25,+0.21$ \\
\hline $1200 \ldots$ & -21.26 & $-0.24,+0.21$ \\
\hline $1400 \ldots$ & -21.31 & $-0.22,+0.20$ \\
\hline $1600 \ldots$ & -21.41 & $-0.22,+0.18$ \\
\hline $2000 \ldots$ & -21.36 & $-0.26,+0.25$ \\
\hline $2400 \ldots$ & -21.51 & $-0.22,+0.27$ \\
\hline
\end{tabular}

a Assumed offset of rest frame to red of $\operatorname{Ly} \alpha$ and $\mathrm{C}$ IV emission-line redshift in $\mathrm{km} \mathrm{s}^{-1}$.

${ }^{b} J_{v}$ is quoted in units of ergs $\mathrm{cm}^{-2} \mathrm{~s}^{-1} \mathrm{~Hz}^{-1}$ $\mathrm{sr}^{-1}$ and refers to the mean background intensity at the Lyman limit.

${ }^{c}$ Quoted errors are the formal $90 \%$ confidence limits determined from $\chi^{2}$ fitting for the case of one interesting parameter.

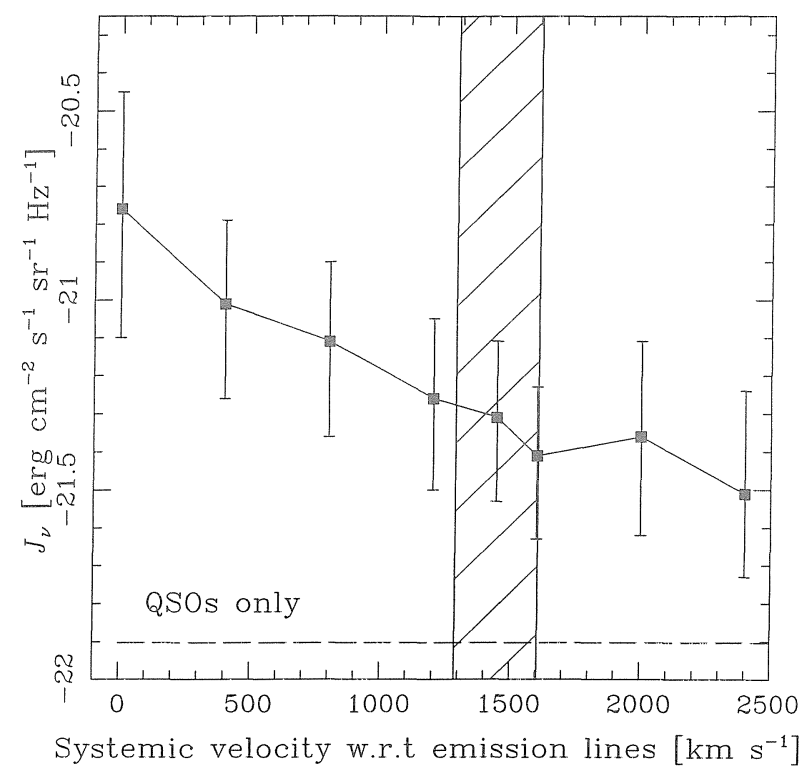

Fig. 1.-Observationally derived values for the intensity of the intergalactic ionizing radiation, $J_{v}$, obtained using the Ly $\alpha$ absorption-line data of Lu et al. (1991). At each plotted point the background intensity has been derived assuming that every QSO emission-line redshift in the sample was offset to the blue of the continuum value by the indicated velocity. The shaded region delimits the range in which the mean velocity shift for the $\mathrm{Lu}$ et al. sample is thought to lie on the basis of measured shifts for other QSOs. The horizontal dashed line indicates the expected background due solely to QSOs in a universe optically thick to ionizing radiation (Madau 1992). Error bars indicate $90 \%$ confidence limits derived from $\chi^{2}$ fitting.

approximation to the Ly $\alpha$ column density distribution (eq. [2]) or to an error in the model assumptions. An error of $\pm 0.1 \mathrm{in}$ the power of the fitting function resulted in $\lesssim 0.15$ error in the derived value of $\log J_{v}$ and this is within the formal errors calculated for our best-guess model of the $\mathrm{Lu}$ et al. data set. Chernomordik \& Ozernoy (1993) have suggested that an exponent of -1.4 , rather than -1.7 , is a more accurate representation of the number-column density relationship given in equation (2). Adopting their value leads to a derived background intensity which agrees with the calculated value given in $\mathrm{M} 92$ within errors (viz., $\log J_{v}=-21.76_{-0.20}^{+0.23}$ ).

More recent work by Meiksin \& Madau (1993) suggests that the opacity of the universe is lower than is assumed in Madau's optically thick models, and this would imply that the contribution of QSOs to the ionizing background is closer to $50 \%$. Evidence supporting the possible dominance of QSO radiation also comes from recent work concerning the number of $\mathrm{Ly} \alpha$ clouds at low redshift (Ikeuchi \& Turner 1991) and revisions to estimates of the space density of QSOs at high redshifts (Boyle 1991).

\section{CONCLUSIONS}

We have presented results in which the influence of an absorbed intergalactic ionizing spectrum and an unabsorbed QSO spectrum on Ly $\alpha$ forest clouds has been studied for the first time. We have also taken the effects of emission-line velocity shifts into account in deriving revised estimates for the strength of the UV ionizing background. If the universe is optically thin, then QSOs alone provide sufficient flux to ionize Lyo clouds and the intergalactic medium. If the universe is optically thick, the importance of QSOs depends on the exact 
value of the opacity. For the optically thick model of M92 and a velocity shift of $\approx 1450 \mathrm{~km} \mathrm{~s}^{-1}$ the calculated contribution of QSOs alone accounts for $\approx 25 \%$ of the required ionizing intensity, but may be as much as $\approx 50 \%$ if the lower opacity models of Meiksin \& Madau (1993) are used. Evidence supporting the possible dominance of QSO radiation also comes from recent work concerning the number of Ly $\alpha$ clouds at low redshift (Ikeuchi \& Turner 1991) and revisions to estimates of the space density of QSOs at high redshifts (Boyle 1991).

An additional feature of emission-line velocity shifts is the explanation of "excess" of clouds close to QSOs relative to the predictions of conventional (zero velocity shift) models. It is thus not necessary to invoke additional mechanisms such as BDO's model involving long-term QSO variability to explain the numbers of these clouds.

An understanding of the nature of emission-line velocity shifts in QSO spectra is important for the correct interpretation of Inverse Effect data. Work is in progress to determine more accurately the nature, and mean value, of broad emission line velocity shifts in a sample of luminous QSOs, and will be reported at a later date (Espey \& Junkkarinen 1993). We believe that it is also necessary to improve the statistics of the near-QSO region through publication of Ly $\alpha$ absorption-line data for regions up to several thousand kilometers per second to the red of $\mathbb{L} y \alpha$ emission rather than conventionally ending the lists at the peak of the emission line.

The author wishes to thank Piero Madau for providing the results of his calculations of the ionizing background, and Gary Ferland for providing a copy of CLOUDY. This analysis was also greatly facilitated by the provision of the Ly $\alpha$ forest data in electronic form by Limin Lu. An impetus for this work came during a visit to Leiden Observatory, and the financial assistance of the Leids Kerkhoven Bosscha Fonds is greatly appreciated. Regina Schulte-Ladbeck is thanked for her critical reading of the original drafts of the manuscript, and the comments of the anonymous referee helped to improve the clarity of presentation.
Alloin, D., Barvainis, R., Gordon, M. A., \& Antonucci, R. R. J. 1992, A\&A, 265, 429

Bajtlik, S., Duncan, R. C., \& Ostriker, J. P. 1988, ApJ, 327, 570 (BDO)

Bechtold, J., Weymann, R. J., Lin, Z., \& Malkan, M. 1987, ApJ, 315, 180

Boyle, B. J. 1991, in Texas-ESO/CERN Symposium on Relativistic Astrophysics, Cosmology and Fundamental Physics, ed. J. D. Barrow, L. Mestel, \& P. A. Thomas (Ann. NY Acad. Sci., Vol. 647), 14

Carswell, R. F., et al. 1991, ApJ, 381, L5

Chernomordik, V. V., \& Ozernoy, L. M. 1993, ApJ, 404, L5

Corbin, M. R. 1990, ApJ, 357, 346

Espey, B. R. 1989, Ph.D. thesis, Cambridge Univ.

Espey, B. R., \& Junkkarinen, V. T. 1993, in preparation

Fall, S. M., \& Pei, Y. C. 1993, ApJ, 402, 479

Ferland, G. J. 1991, HAZY, a Brief Introduction to CLOUDY, OSU Astron.

Dept. Internal Rep. 91-01

\section{REFERENCES}

Gaskell, C. M. 1982, ApJ, 263, 79

Hutchings, J. B., Gower, A. C., \& Price, R. 1987, AJ, 92, 6

Ikeuchi, S., \& Turner, E. L. 1991, ApJ, 281, L1

Lu, L., Wolfe, A. M., \& Turnshek, D. A. 1991, ApJ, 367, 1

Madau, P. 1992, ApJ, 389, L1 (M92)

Meiksin, A., \& Madau, P. 1993, ApJ, in press

Murdoch, H. S., Hunstead, R. W., Pettini, M., \& Blades, J. C. 1986, ApJ, 309, 19

Tytler, D., \& Fan, X.-M. 1992, ApJS, 79, 1

Wilkes, B. J. 1984, MNRAS, 207, 73

. 1986, MNRAS, 218, 331

Wilson, A. S., \& Heckman, T. M. 1985, in Astrophysics of Active Galaxies and Quasi-stellar Objects, ed. J. S. Miller (Mill Valley: University Science Books) 